\title{
3D-Printed Vision-Based Micro-Force Sensor Dedicated to In Situ SEM Measurements
}

\author{
Valérian Guelpa ${ }^{1}$, Jean-Sébastien $\operatorname{Prax}^{1}$, Youen Vitry ${ }^{2,3}$, Olivier Lehmann ${ }^{1}$, Sam Dehaeck ${ }^{3}$, \\ Patrick Sandoz ${ }^{4}$, Cédric Clévy ${ }^{1}$, Nadine Le Fort-Piat ${ }^{1}$, Pierre Lambert ${ }^{2}$ and Guillaume J. Laurent ${ }^{1}$
}

\begin{abstract}
Working at micro-scale efficiently requires accurate and integrated force feedback implemented with a sensor adapted to the scale. This paper presents a 3D-printed vision-based micro-force sensor intended to be used inside the chamber of a Scanning Electron Microscope (SEM). The combination of 3D printed elastic structures with a highly effective vision based measurement method allows to design integrated sensors at the cutting edge of the state of the art. Moreover the presented design respects the Abbe's alignment principle. The paper presents the general design, manufacturing and experimental characterization in SEM environment of the proposed sensor. Images of periodical patterns are used to measure the differential displacement between the two parts of the compliant structure. By the knowledge of its stiffness, the force applied on it is measured. The stiffness of the elastic structure has been measured to be $15.3 \mathrm{~N} \cdot \mathrm{m}^{-1}$, leading to a force range of $25 \mu \mathrm{N}$.
\end{abstract}

\section{INTRODUCTION}

Efficiently working at micro-scale is a difficult task because of fast dynamics, surface forces and more generally unfavourable scaling factor. Despite the important previous work, there is still a lack of models and knowledge to estimate and quantify their influence. So it is often required to implement accurate force feedback during experiments. In this way several teams investigated the integration of force sensors, wishing them smaller and closer to the contact.

Most of microforce sensors are based on monolithic elastic microstructures such as cantilevers or beams. Different physical principles have been proposed to measure the position or the deformation of the structure: capacitive [1], [2], [3], piezoresistive [4], [5], [6], strain gauges [7], [8], magnetic [9], and optical [10], [11]. However the need for resolution often goes with the design of a low-stiffness compliant mechanism at the expense of dynamics. In most cases, the displacement sensing part of the force sensor is also bulky (see Fig. 1) and can not be placed close to the contact point, making the measurement potentially inaccurate. Moreover only a very few of these devices respect the Abbe's alignment principle [12]: whereas the best is to have on the same line (corresponding to the sensitive direction) both the force

\footnotetext{
1 Automation and Micro-Mechatronics Systems Department, FEMTO-ST Institute, Univ. Bourgogne Franche-Comté, UMR CNRS 6174, ENSMM, Besancon, France

2 Université libre de Bruxelles, BEAMS dpt, CP 165/56, 50 avenue Roosevelt, B-1050 Brussels

3 Université libre de Bruxelles, TIPs dpt, CP 165/67, 50 avenue Roosevelt, B-1050 Brussels

4 Department of Applied Mechanics, FEMTO-ST Institute, Univ. Bourgogne Franche-Comté, UMR CNRS 6174, ENSMM, Besancon, France
}

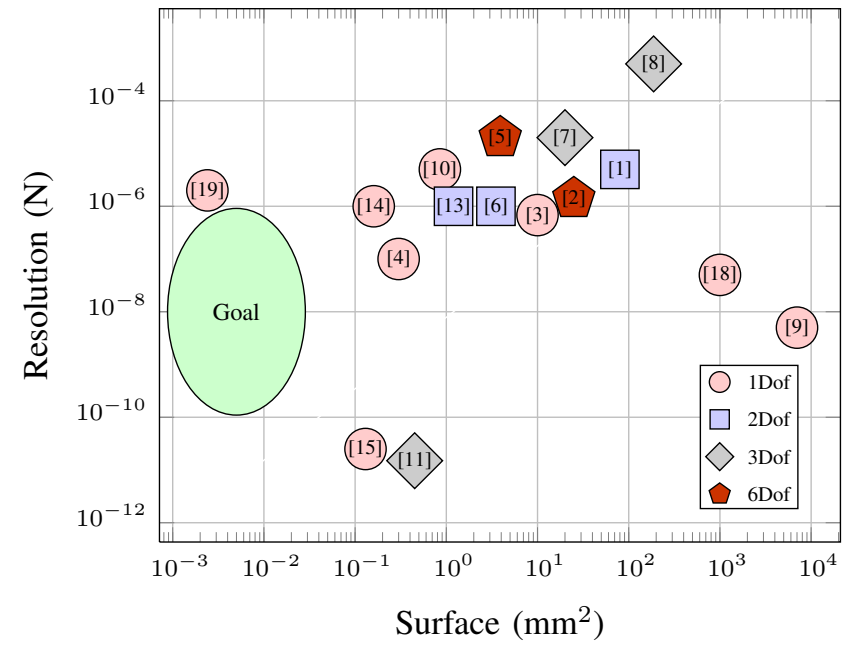

Fig. 1. Resolution of micro-force sensors mentioned in this paper, according to their surface.

application point and the sensing axis, it is often difficult to follow this rule for the design of micro-sensors.

Some visual-based approaches have been developed to measure the force deforming an elastic microstructure by measuring its displacement [13], [14]. Vision as a force measurement tool has some advantages such as the absence of contact with the compliant mechanism as well as the possibility of measurements along several axes. However these methods are usually limited by the resolution of the optical sensor (ie number of pixels) and by the measurement method used (point detection, correlation, model-based tracking, etc.). Nevertheless it is possible to investigate periodic pattern-based approaches to improve the resolution of visual-based sensing drastically [15], [16], [17]. In previous work [18], we applied such approach to force sensing. To date, we obtained a resolution below $50 \mathrm{nN}$ with a centimeter-sized compliant structure with a stiffness of $240 \mathrm{~N} . \mathrm{m}^{-1}$ and a $10 \times$ optical microscope.

Recently, 3D-printing methods have been exploited to process compliant end-effectors used as force sensors by means of vision [19]. Indeed, recent technological advances enable us to consider a new step in the use of vision for micro-force measurements. The dip-in laser lithography is known as an efficient rapid-prototyping technique and is now usable at micro-scale thanks to the two-photon absorption process

In this paper, we propose to develop a sensor combining the performances of pattern-based approaches to the inte- 
gration capacities at microscale of 3D-printing. The sensor is also dedicated to be used inside a Scanning Electron Microscope (SEM), a challenging context for force measurement because of the limited space, the restricted number of effective measurement principles and the limited quality of vision.

The proposed visual-based micro-force sensor addresses three objectives:

- to integrate the micro-sensor (resolution better than $1 \mu \mathrm{N}$, size smaller than $100 \mu \mathrm{m}$, see the goals in Fig. 1), including closeness of the sensing part to the contact point and respect of Abbe's alignment principle;

- to investigate the suitability of a 3D-printed sensor;

- to operate inside the chamber of a scanning electron microscope (SEM).

The paper is organized as follows. Section II describes the sensor concept from it mechanical design to the integration of the patterns. Section III details the mechanical design of the compliant structure. Section IV presents the fabrication process and the experimental validation of the sensor. The last section concludes the paper.

\section{VISUAL-BASED FORCE SENSING AT THE MICROSCALE}

The concept of micro-force sensor further presented allows to measure passively and directly in the direction of the applied force, allowing the respect of the Abbe's alignment principle. In this section we will briefly introduce the flexure mechanism and the method used to obtain displacement measurement based on visual feedback.

\section{A. Flexure systems}

To measure the force only applied along one direction, so only the displacement along one direction, the structure of the sensor have to guide the movement. The simplest possible guidance for linear motion consists in using two straight plates (Fig. 2a). To avoid possible mechanical instabilities in the thin leaf springs and be closer to ideal kinematics of a parallelogram, four clamped plates can be used (Fig. 2b). Instead, a third solution uses four notch hinges (Fig. 2c), even more closer to ideal kinematics of a parallelogram. The design with four notch hinges better defines the centers of rotation in the kinematics, while it concentrates larger mechanical stresses to these points. In the present case, the material is defined by the dip-in laser lithography process, like SU-8 resin. Since this material has good elastic limit, the design with four clamped plates is preferable.

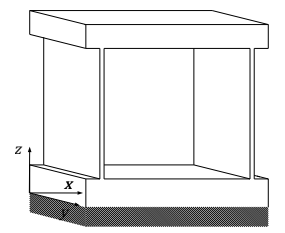

(a) Leaf-spring linear guidance
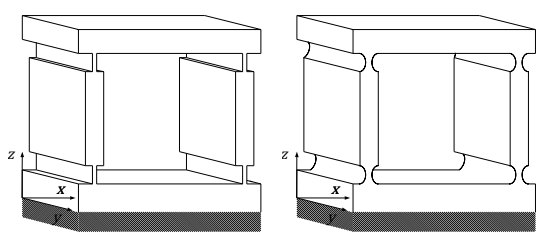

(b) Fou plates

clamped (c) Four notch hinges

Fig. 2. The three considered flexures.
The stiffnesses of basic flexures are well known and can be calculated with the plate theory of the continuum mechanics [20], [21]. They consist of four clamped plates linking a moving part to a non-moving part with an intermediate rigid body, thus making a leaf spring (see Fig. 3). According to [20], the stiffness $K$ is given by:

$$
K=\frac{1}{\xi\left(\xi^{2}-3 \xi+3\right)} \frac{2 E b h^{3}}{l^{3}}
$$

with $b, h$ and $l$ the main dimensions (see Fig. 3.a) of the flexure, $\xi=\frac{2 l_{c}}{l}$ the ratio between the length of the two clamped plates and the total length of the spring (a standard value is 0.3 ), $E$ being the Young Modulus of the material and $I$ being the moment of inertia (which is $\frac{b h^{3}}{12}$ here).

a)
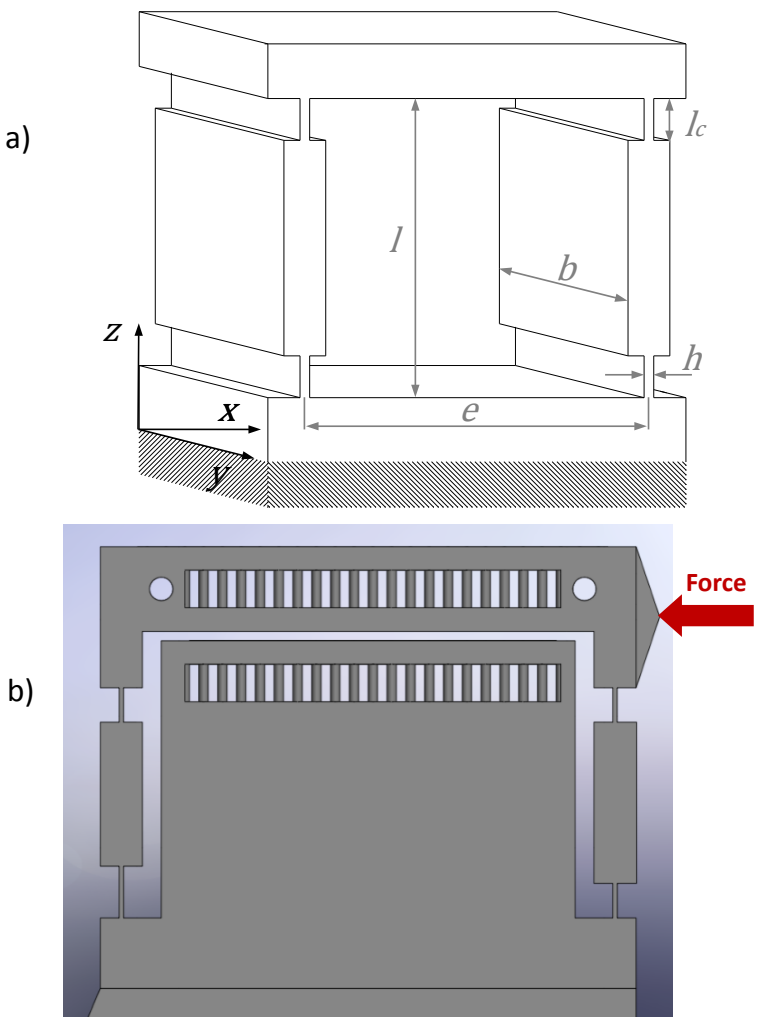

Fig. 3. a) Sketch of the concept for the mechanism, b) first CAD model of the mechanism. The non-moving part of the leaf spring acts as a mechanical stop and as reference for the differential measurement.

If a force $F$ is applied on the mechanism, the moving part will move in the direction of application. The restoring force of a spring simply gives the force depending on the stiffness and the displacement of the moving part:

$$
F=K \delta
$$

with $\delta$ the displacement of the moving part when a force $F$ is applied at its tip along $\mathrm{X}$-axis.

\section{B. Phase-difference visual measurement}

The use of a SEM as a metrological tool is difficult: images are noised, especially by high frequencies disturbances, and they suffer from drift with time. However a frequency-based 
method to measure the displacement of a moving part by vision exists that could solve most of the noise problems on the spectral domain. It consists in using periodical patterns like a twin set of stripes with the same period (see Fig. 6). One is positioned on the moving part while the other is on the mechanical stop. These two patterns allow a differential measurement, a need for force measurement that reduces at the same time the impact of image drift [18].

The image processing aims to measure the phase shift that is created between the two sets of stripes with a displacement of the moving part.

This phase shift is related to the displacement by a scalar product in the frequency domain:

$$
\mathscr{F}(f(x-\delta))=e^{-2 \pi i \delta \xi} \cdot \mathscr{F}(f(x))
$$

where $\mathscr{F}$ is the Fourier transformation, $f(x)$ is a space function, $x$ the coordinate along the axis, $\delta$ the displacement and $\xi$ the reciprocal of $x$. In this way, a displacement $\delta$ of the target induces a phase shift $\Delta \Phi$ in the frequency domain:

$$
\Delta \Phi=2 \pi \delta \xi
$$

A single-frequency spectral component - corresponding to the spatial frequency of the periodic pattern used - is computed instead of performing a complete Discrete Fourier Transform (DFT) to improve the computation times. For that purpose a complex analysis vector $Z(k)$ is used. It is defined by a Gaussian window and a periodic signal at the period $P$ of the stripe set (in pixels):

$$
Z(k)=e^{-\left(\frac{k-N / 2}{N / 4.5}\right)^{2}} \cdot e^{-\left(\frac{2 i \pi(k-N / 2)}{P}\right)}
$$

with $k$ the pixel index and $N$ the image width in pixels. The phase $\Phi$ is then given by the argument of the dot product between vector $Z$ and the vector of pixel intensities.

The phase shift can then be easily converted into the length of the displacement $\delta$ of the moving part with:

$$
\delta=\frac{\Delta \Phi L}{2 \pi}+m L
$$

where $L$ is the period of the periodic pattern in meters, $\Delta \Phi$ the phase shift between the sets of stripes and $m$ an integer. It is an ambiguous measure since when the displacement equals a multiple of the period, the phase shift returns to zero due to the periodicity. Thus, this method gives the displacement modulo $L$. In this paper, it does not cause any problem since the sensor is designed to allow a displacement under or equal to the period but not above.

\section{SENSOR DESIGN}

\section{A. Determination of the main stiffness}

3D printing shows good capabilities to produce complex shapes at micro-scale. Trials show that a period of $4 \mu \mathrm{m}$ can be obtained through techniques of dip-in laser lithography with a Photonic Professional GT device from Nanoscribe. Displacement can be measured with a resolution of $1 / 10000^{t h}$ of this period (see [18]), provided that approximately 20 periods are visible (to work on a reasonable number of periods, to have a satisfying information redundancy) so for $e>80 \mu \mathrm{m}$. Thus the theoretical smallest measurable displacement of the moving part would be $400 \mathrm{pm}$.

To be able to test our method of force sensing with standard capacitive force sensors, we set ourselves on the objective of producing a sensor with a resolution of $1 \mathrm{nN}$. Indeed the equation (2) gives then the desired stiffness of the flexure: $K=2.5 \mathrm{~N} . \mathrm{m}^{-1}$.

The IP-Dip resin used during the 3D-printing process is assumed to have similar mechanical properties as the SU8. Thus, the device is designed considering the mechanical properties of SU-8 resin: the Young modulus considered is $E=2 \mathrm{GPa}$ and the yield strength is $\sigma_{Y}=34 \mathrm{MPa}$.

\section{B. Ranges of the Parameters}

To achieve this stiffness, the length of the flexure could be calculated now as a function of its width and its thickness through the equation (1):

$$
l=\left(\frac{2 E b h^{3}}{K \xi\left(\xi^{2}-3 \xi+3\right)}\right)^{1 / 3}
$$

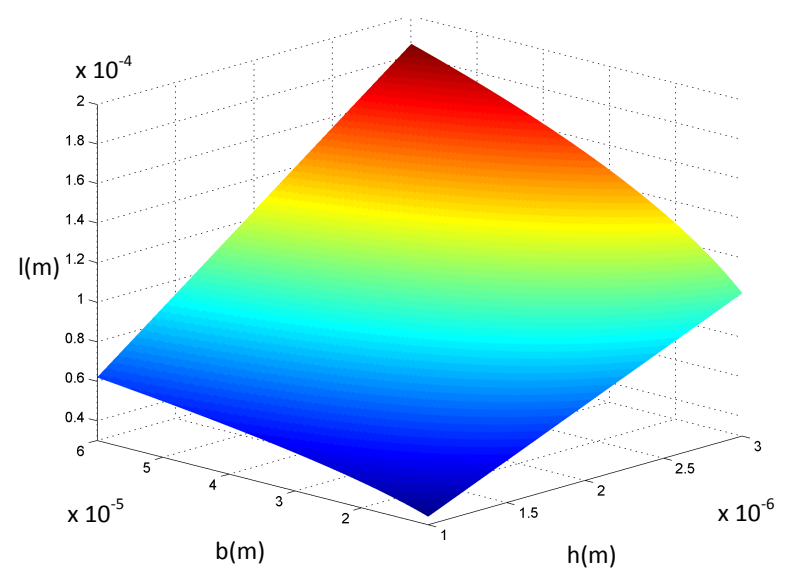

Fig. 4. Length $l$ of the mechanism as a function of its width $b$ and thickness $h$ for $K=2.5 \mathrm{~N} \cdot \mathrm{m}^{-1}$.

This function is plotted in Fig. 4 for a thickness $h$ varying from $1 \mu \mathrm{m}$ to $3 \mu \mathrm{m}$ and a width $b$ varying from $10 \mu \mathrm{m}$ to $60 \mu \mathrm{m}$.

In Fig. 4 all the values of $l$ are displayed but not all of them are realistic, so some conditions were added. The first condition comes from the plate theory on which calculations are based: $b \geq 10 h$ and $l \geq 10 h$. Thus the right corner of the surface is removed. It is also important to ensure that the mechanism will not break. Since a mechanical stop was added to the mechanism to prevent the displacement to go beyond the length of one period (which is the range of the unambiguous displacement measurement), the only need is to ensure that the greatest displacement before mechanical break is greater than $4 \mu \mathrm{m}$. The formula for the largest displacement for the mechanism is:

$$
\delta_{\max }=\frac{l^{2} \sigma_{Y}}{3 E h}
$$




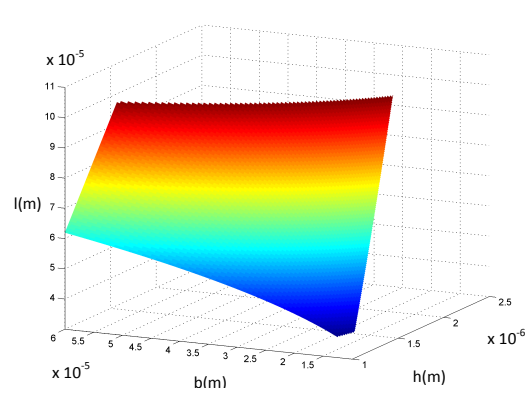

(a) Allowed domain.

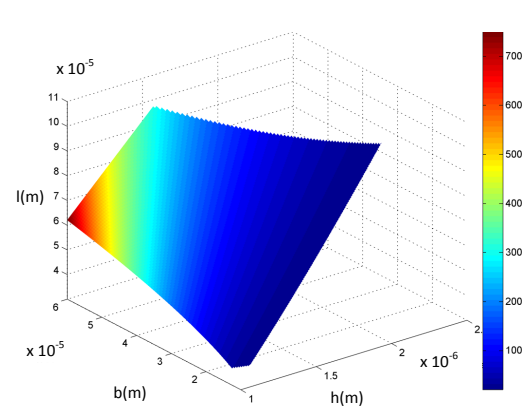

(d) Linear stiffness $K_{\alpha x}$

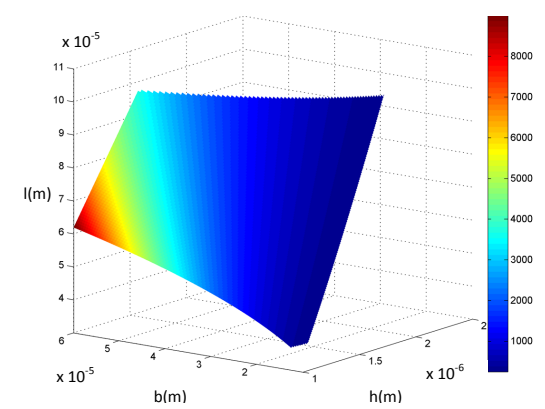

(b) Linear stiffness $K_{y}$

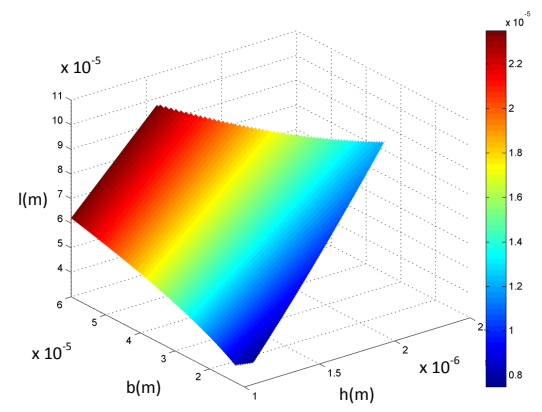

(e) Linear stiffness $K_{\alpha y}$

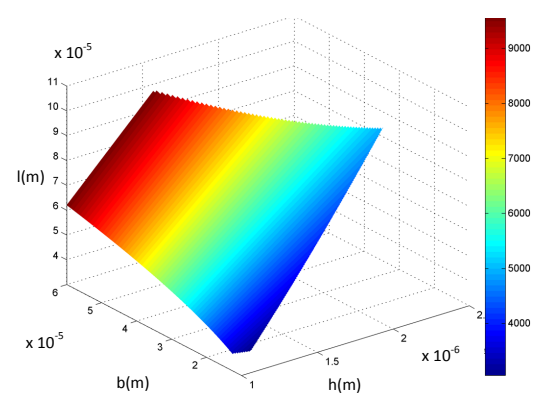

(c) Linear stiffness $K_{z}$

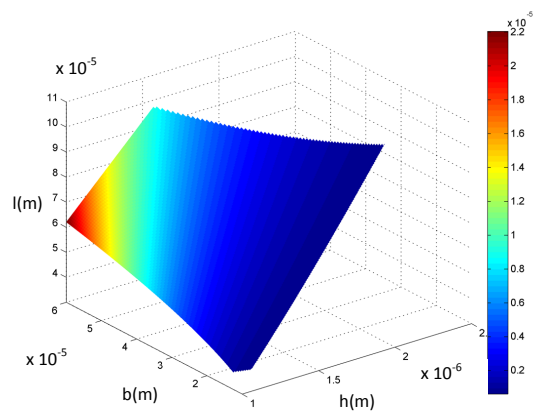

(f) Linear stiffness $K_{\alpha z}$

Fig. 5. Analysis of the variations of linear stiffnesses $K_{y}, K_{z}, K_{\alpha x}, K_{\alpha y}$ and $K_{\alpha z}$ in the domain of all the geometric parameters once all the criteria are taken into account.

All the calculations before only take into account a force applied in the direction of the $x$ axis (the axes are shown in Fig. 3). But even in this case, forces of traction and compression are also applied on the compliant plates in the $z$ axis unless the force on the $x$ axis is applied at a distance of $l / 2$, in which case these unwanted forces negate themselves. This phenomenon can be neglected in a first approximation; however according to [20] we chose to ensure the validity of this approximation by limiting the ratio $l / e$, thus diminishing these forces. A maximum ratio of 1 is a reasonable choice, meaning that the plates cannot be longer than the distance between them. This criterion greatly reduces the ranges of the usable geometric parameters. Fig. 5.a shows the possible dimensions for our mechanism.

\section{Selection of the Parameters}

The mechanism has to allow only a movement along the $x$ axis and blocks the others. To do that, the selected parameters are those which give the highest stiffnesses possible along the other axes. The different stiffnesses are as follow:

$$
\begin{gathered}
K_{y}=\frac{2 E h b^{3}}{l^{3} \xi\left(\xi^{2}-3 \xi+3\right)} \\
K_{z}=\frac{2 E h b}{l \xi} \quad(10) \quad K_{\alpha x}=\frac{2 E h b^{3}}{12 l \xi} \\
K_{\alpha y}=\frac{E h b^{3}}{6 l \xi}+2\left(\frac{e}{2}\right)^{2} \frac{E h b}{l \xi} \\
K_{\alpha z}=\frac{G b h^{3}}{6 l \xi}+2\left(\frac{e}{2}\right)^{2} \frac{E h b^{3}}{l^{3} \xi}
\end{gathered}
$$

with $G$ being $\frac{E}{2(1+\nu)}$, the shear modulus, and $\nu$ being the Poisson ratio (0.33 in this case). $K_{x}, K_{y}$, and $K_{z}$ are the linear stiffnesses (in N.m ${ }^{-1}$ ) of the mechanism respectively along the axes $x, y$, and $z$; since $K_{x}$ is the linear stiffness along the axis of the periodical patterns it has been referred to as simply $K . K_{\alpha x}, K_{\alpha y}$, and $K_{\alpha z}$ are the angular stiffnesses (in N.m.rad ${ }^{-1}$ ) of the mechanism respectively around the axes $x, y$, and $z$. Their values are shown in the Fig. 5 except for $K\left(\right.$ or $\left.K_{x}\right)$ which has already been set at $2.5 \mathrm{~N} . \mathrm{m}^{-1}$. It appears clearly that the left corner of the surface, with a maximal value of $b$ and a minimal value of $h$, maximizes all the stiffnesses. The chosen dimensions are presented in Table I.

\section{TABLE I}

OVERVIEW OF THE DIMENSIONS AND MAIN STIFFNESS OF THE VISUAL-BASED SENSOR.

\begin{tabular}{c|cccccc}
\hline & $L(\mu \mathrm{m})$ & $h(\mu \mathrm{m})$ & $b(\mu \mathrm{m})$ & $l(\mu \mathrm{m})$ & $l_{c}(\mu \mathrm{m})$ & $\mathrm{K}\left(\mathrm{N} \cdot \mathrm{m}^{-1}\right)$ \\
\hline Theoretical & 4 & 1 & 49.3 & 49 & 7.4 & 2.5 \\
Actual size & 4 & 1.5 & 51 & 47 & 7 & 10.1 \\
Characterized & & & & & & 15.3 \\
\hline
\end{tabular}

\section{EXPERIMENTAL RESULTS}

\section{A. Processing}

As planned during the design step, the structure was written using dip-in laser lithography with a Nanoscribe Photonic Professional GT. The $63 \times$ objective and IP-Dip 
photoresist were used for printing, which allowed for the highest resolution $(<1 \mu \mathrm{m})$ when writing 3D structures. As the final sensor size was well above the largest block size that could be written with the galvo-scanner (i.e. larger than $150 \times 150 \mu \mathrm{m}^{2}$ in a horizontal plane), the object was divided in multiple blocks by the Nanoscribe Describe software. To speed up the process, non-critical supporting blocks needed to attach the sensor to the robotic platform, were printed in shell and scaffold mode and fully cured later with a flood UVillumination. The critical block containing the force sensor was written in solid mode. The result can be seen in Fig. 6 and 7 .

However the high requirement level initially expected is difficult to achieve. The setup of the Fig. 6 does not respect the theoretical dimensions. The comparison of what we expect and what we obtained is made in Table I. The two main reasons are the size of the voxel used for 3D-prining and the resin retractation during polymerization. We can see that even if practical dimensions are only slightly different from theoretical, the deducted stiffness is multiplied by 4 , reaching 10.1 N.m ${ }^{-1}$.

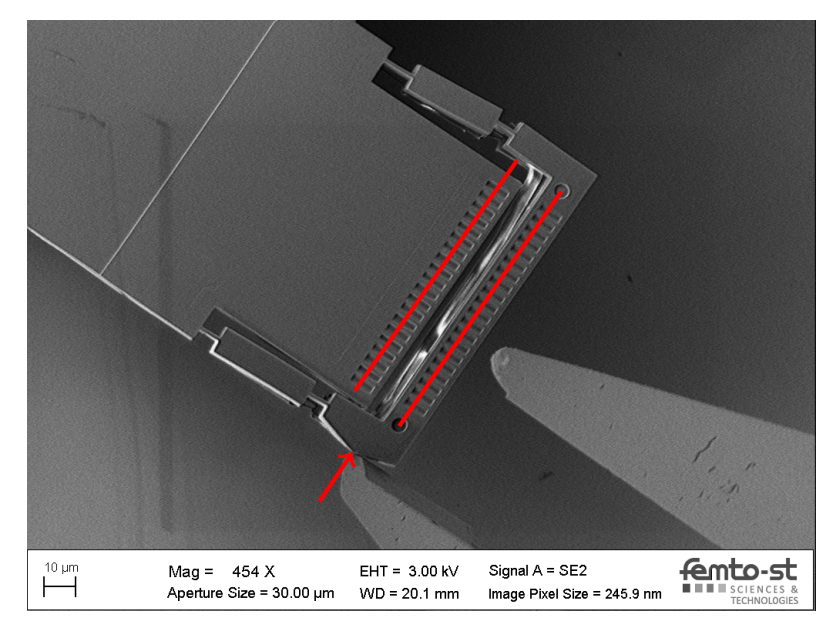

Fig. 6. SEM image of the sensor during experiment. Measurement are done with similar videos or images.
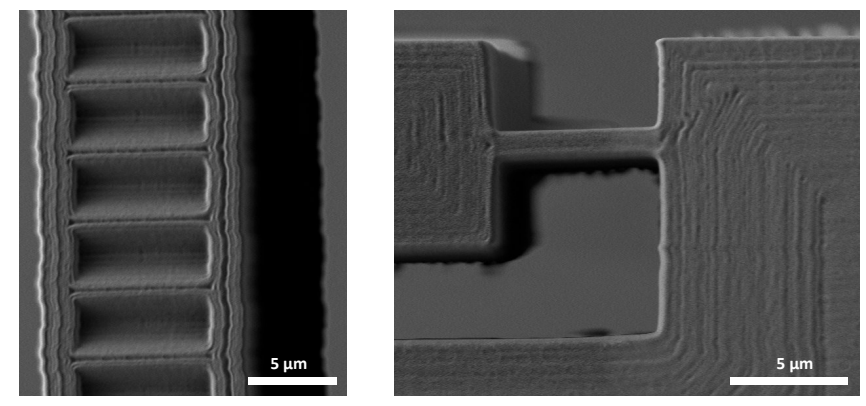

Fig. 7. SEM images of two critical points of the sensor: its periodic grid and one of its compliant plate.

\section{B. Stiffness and trueness evaluation}

This section deals with the identification of the real stiffness of the compliant mechanism and aims to proof the feasibility of the concept. The sensor was tested under SEM and its measures compared with a capacitive sensor, used as reference.

The visual-based sensor is fixed to a first robotic platform of the SEM. A micro-gripper (FT-G32 from FemtoTools) is controlled by a second robot. Its instrumented finger is used as the reference sensor (see Fig. 6).

A back and forth experiment was completed to evaluate the trueness and the stiffness of the setup. Fig. 8 presents the force measured by the capacitive sensor relatively to the differential displacement measured by vision during three cycles. It allows to evaluate the stiffness of the setup as 15.3 N.m ${ }^{-1}$. This evaluated stiffness is different from the expected value $\left(K=10.1 \mathrm{~N} . \mathrm{m}^{-1}\right)$. It could be put down to the imperfections due to the processing of the setup, on the upper bound of what can be done with dip-in laser lithography methods, and to the slightly difference of young modulus and shear modulus between SU-8 and IP-Dip.

Fig. 9 illustrates the resulting force measurements by vision and by the capacitive sensor. The trueness, taken as the standard deviation of the error, is equal to $0.7 \mu \mathrm{N}$ on a global range of $25 \mu \mathrm{N}$. The two force measurements are well-correlated.

The principal limitation of the method is the important stretch even within each image due to the limited scanning speed of the SEM. It often induces a distortion of the image during movements, making a high-resolute measure difficult to obtain. In this way an important improvement of the method could be to reduce this deviation by a better control of the scan, as proposed for example by [22]. Here only two lines are necessary to do the measurement, reducing drastically the scan time and therefore improving the trueness in return for a smaller field of view.

\section{CONCLUSION}

A new design of passive micro-force sensor by vision is presented. The chosen design is the result of a full mechanical study driven by the wish of effective integration. The method benefits from the advantages of dip-in laser lithography (fast prototyping, monolithic piece, low cost) and from the performances provided by the use of periodical patterns (nanometric measurement of position). The result is a promising highly integrated sensor, with a small size compared to most of other sensors (see Fig. 1).

The trueness was experimentally evaluated to be $0.7 \mu \mathrm{N}$ with a capacitive sensor as reference on a global range of $25 \mu \mathrm{N}$. The final stiffness of $15.3 \mathrm{~N} . \mathrm{m}^{-1}$ is higher than expected, but the calibration step allows to take it into account for futur designs: a flexure with the required stiffness of $2.5 \mathrm{~N} . \mathrm{m}^{-1}$ could be process iteratively, benefiting from the timeliness of 3D-printing process.

The next goal is to improve the resolution while remaining small size, mainly by improving the image quality given 
by the SEM. Next works will also concern the use of two sensors as end-effectors in one gripper to implement manipulating tasks in SEM without any additional force sensor. The extension to force measurements along more degrees of freedom could also be considered.

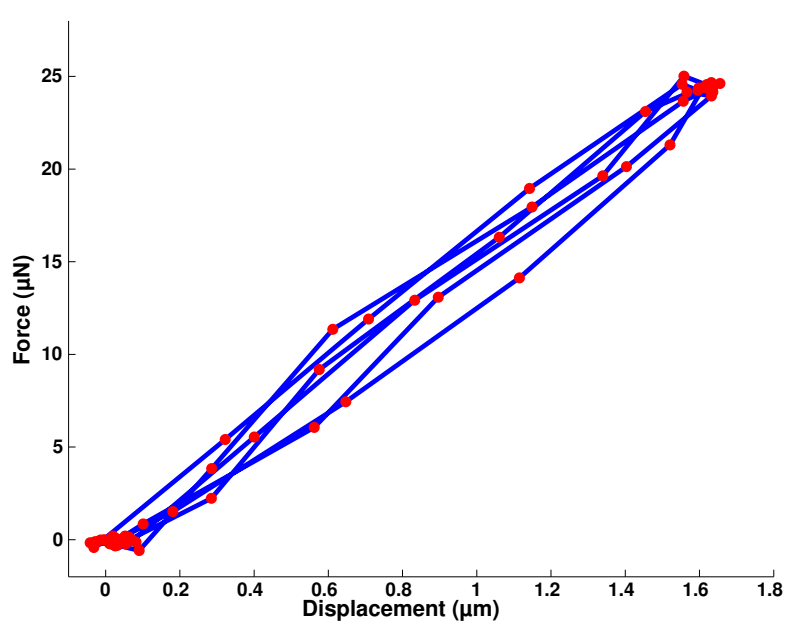

Fig. 8. Force measured by capacitive sensor relatively to position measurement by vision. Thus the stiffness is evaluated as $15.3 \mathrm{~N} \cdot \mathrm{m}^{-1}$.

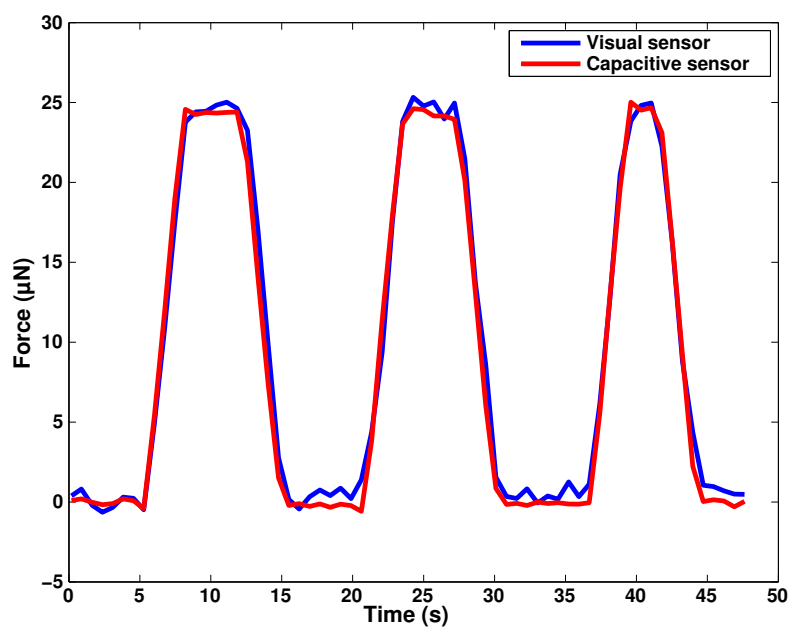

Fig. 9. Force measured by the capacitive sensor and by vision, relatively to the time. The trueness ( $\sigma$ of the error) is measured as $0.7 \mu \mathrm{N}$.

\section{ACKNOWLEDGMENT}

This work has been supported by Région de Bourgogne Franche-Comté, Labex ACTION (ANR-11-LABX-01-01) and Equipex ROBOTEX (ANR-10-EQPX-44-01). This work has been partially supported by the IAP 7/38 MicroMAST funded by BELSPO, the FNRS grant GEQ 22687275 and ULB.

\section{REFERENCES}

[1] R. J. Wood, K.-J. Cho, and K. Hoffman, "A Novel Multi-Axis Force Sensor for Microrobotics Applications," Smart Materials and Structures, vol. 18, 2009.
[2] F. Beyeler, S. Muntwyler, and B. J. Nelson, "A Six-Axis MEMS Force and Torque Sensor With Micro-Newton and Nano-Newtonmeter Resolution," J. of Microelectromecanical Systems, vol. 18, no. 2, pp. 433-441, 2009.

[3] Y. Sun, S. N. Fry, D. P. Potasek, D. J. Bell, and B. J. Nelson, "Characterizing Fruit Fly Flight Behavior Using a Microforce Sensor With a New Comb-Drive Configuration," J. of Microelectromechanical Systems, vol. 14, no. 1, pp. 4-11, 2005.

[4] B. Komati, J. Agnus, C. Clevy, and P. Lutz, "Prototyping of a Highly Performant and Integrated Piezoresistive Force Sensor for Microscale Applications," J. of Micromechanics and Microengineering, vol. 24, 2014.

[5] P. Estevez, J. Bank, M. Porta, J. Wei, P. Sarro, M. Tichem, and U. Staufer, "6 DOF Force and Torque Sensor for Micro-Manipulation Applications," Procedia Engineering, vol. 25, pp. 39-42, 2011.

[6] M. Billot, X. Xu, J. Agnus, E. Piat, and P. Stempflé, "Multi-axis mems force sensor for measuring friction components involved in dexterous micromanipulation: design and optimisation," Int. J. of Nanomanufacturing, vol. 11, no. 3-4, pp. 161-184, 2015.

[7] K. Kristiansen, P. McGuiggan, G. Carver, C. Meinhart, and J. Israelachvili, "3D Force and Displacement Sensor for SFA and AFM Measurements," Langmuir, vol. 24, pp. 1541-1549, 2008.

[8] P. J. Berkelman, L. L. Whitcomb, R. H. Taylor, and P. Jensen, "A Miniature Microsurgical Instrument Tip Force Sensor for Enhanced Force Feedback During Robot-Assisted Manipulation," IEEE Trans. on Robotics and Automation, vol. 19, no. 5, pp. 917-922, 2003.

[9] J. Abadie, E. Piat, S. Oster, and M. Boukallel, "Modeling and Experimentation of a Passive Low Frequency Nanoforce Sensor Based on Diamagnetic Levitation," Sensors and Actuators A: Physical, vol. 173, pp. 227-237, 2012.

[10] X. J. Zhang, S. Zappe, R. W. Bernstein, O. Sahin, C. C. Chen, M. Fish, M. P. Scott, and O. Solgaard, "Micromachined Silicon Force Sensor Based on Diffractive Optical Encoders for Characterization of Microinjection," Sensors and Actuators A: Physical, 2004.

[11] M. Dienwiebel, E. De Kuyper, L. Crama, and J. W. M. Frenken, "Design and Performance of a High-Resolution Frictional Force Microscope with Quantitative Three-Dimensional Force Sensitivity," Review of Scientific Instrument, vol. 76, 2005.

[12] G. X. Zhang, "A study on the Abbe principle and Abbe error," CIRP Annals-Manufacturing Technology, vol. 38(1), pp. 525-528, 1989.

[13] D. J. Cappelleri, G. Piazza, and V. Kumar, "A two dimensional vision-based force sensor for microrobotic applications," Sensors and Actuators A: Physical, vol. 171, no. 2, pp. 340-351, 2011.

[14] R. J. Chang, C. C. Shiu, and C. C. Y., "Self-Biased-SMA Drive PU Microgripper with Force Sensing in Visual Servo," I. J. of Advanced Robotic Systems, vol. 10, 2013.

[15] C. Yamahata, E. Sarajlic, L. Jalabert, M. Kumemura, D. Collard, and H. Fujita, "Mechanical characterization of biomolecules in liquid using silicon tweezers with subnanonewton resolution," in IEEE 22nd Int. Conf. on Micro Electro Mechanical Systems. IEEE, 2009, pp. 607610.

[16] H. Sugiura, S. Sakuma, M. Kaneko, and F. Arai, "On-chip method to measure mechanical characteristics of a single cell by using moiré fringe," Micromachines, vol. 6, no. 6, pp. 660-673, 2015.

[17] V. Guelpa, G. J. Laurent, P. Sandoz, J. G. Zea, and C. Clevy, "Subpixelic Measurement of Large 1D Displacements: Principle, Processing Algorithms, Performances and Software," Sensors, vol. 14, no. 3, pp. 5056-5073, 2014.

[18] V. Guelpa, G. J. Laurent, P. Sandoz, and C. Clévy, "Vision-based microforce measurement with a large range-to-resolution ratio using a twin-scale pattern," IEEE/ASME Trans. on Mechatronics, vol. 20, no. 6, pp. 3148-3156, 2015.

[19] M. Power and G. Z. Yang, "Direct laser written passive micromanipulator end-effector for compliant object manipulation," in IEEE/RSJ Int. Conf. on Intelligent Robots and Systems (IROS), 2015, pp. 790-797.

[20] S. Henein, Conception des guidages flexibles. Lausanne, Switzerland: Presses Polytechniques Universitaires Romandes, 2001.

[21] Y. Bellouard, Microrobotics: Methods and Applications. Boca Raton, Florida: CRC Press, 2009.

[22] T. Tiemerding, C. Diederichs, S. Zimmermann, and S. Fatikow, "Closing the loop: high-speed visual servoing and control of a commercial nanostage inside the SEM," in Manipulation, Manufacturing and Measurement on the Nanoscale (3M-NANO), 2013, pp. 790-797. 